\title{
La aplicación de criterios prudentes en la valoración de activos, costos, gastos y pasivos
}

María Amparo Pachón Pachón ${ }^{1}$

Pachón P., M. A. (2010). La aplicación de criterios prudentes en la valoración de activos, costos, gastos y pasivos. Revista Activos, 14, 161-171.

Recibido: 15 de agosto de 2009 Aceptado: 15 de septiembre de 2009

\section{Resumen}

El documento presenta una amplia caracterización de los criterios técnicos que deben tenerse en cuenta para el reconocimiento de las partidas contables, y especialmente sobre el tratamiento de las contingencias. Recoge los principales argumentos que se presentan en la valoración de partidas contingentes y rescata los criterios de conservadurismo que son necesarios para el registro de los hechos económicos.

Palabras clave: causación, contingencias, realización, hecho económico, reconocimiento, prudencia, conservadurismo.

Pachón P., M. A. (2010). The application of prudent criteria in the evaluation of assets, costs, expenses and debits. Activos Review, 14, 161-171.

\section{Abstract}

The document presents a wide characterization of the technical criteria that must be born in mind for the recognition of the countable topics, and especially on the treatment of 
ACTIVOS | REVISTA DE LA FACULTAD DE CONTADURía PÚblica

the risks, presents the principal arguments that appear in the evaluation of contingent games and it rescues the criteria of conservatism that are necessary for the record of the economic facts.

Key words: Causation, Risks, Achievement, Economic Fact, Recognition, Prudence, Conservatism.

Pachón P., M. A. (2010). L’application de criteres prudents dans l'evaluation d'actifs, de couts, de frais et de passifs. Revue Activos, 14, Pag. (xxx)

\section{Résumé}

Le document présente une vaste caractérisation des critères techniques qui doivent être tenus en compte pour la reconnaissance des départs comptables, et spécialement sur le traitement de l'éventualité, présente les arguments principaux qui se présentent dans lévaluation de départs contingents et il sauve les critères du conservatisme qui sont nécessaires pour le registre des faits économiques.

Mots clé: Caution, une éventualité, une réalisation, un fait économique, une reconnaissance, une prudence, le conservatisme. 


\section{Introducción}

En desarrollo del objeto social, las compañías realizan diferentes transacciones, muchas de las cuales son hechos económicos que afectan la riqueza ${ }^{2}$ y la rentabilidad ${ }^{3}$ del ente económico. Estos hechos económicos se reconocen y acumulan contablemente, teniendo en cuenta las premisas de causación, realización, medición, asignación, materialidad, entre otras, como también el criterio de prudencia, con el fin de determinar la situación financiera de la compañía y el desempeño de la misma, de manera periódica.

La aplicación de las premisas de realización, medición y asignación, presenta algún grado de complejidad, ya que éstas interactúan con la premisa de prudencia, la cual implica el uso de un criterio subjetivo por parte de quien evalúa la transacción y los hechos que lo rodean, afectando la razonabilidad contable.

\section{Premisas para el reconocimiento de los hechos económicos}

La causación implica reconocer los hechos económicos cuando se realizan, es decir, cuando ocurren o existe alta probabilidad de ocurrencia, y no sólo cuando hay movimientos de efectivo. Aplicando un criterio prudente, habrá que causar provisiones para activos, valorizaciones o desvalorizaciones de activos, pasivos estimados y pasivos contingentes.

La realización se relaciona con el momento y las condiciones que se deben dar para que se entienda recibido un activo, incurrido en un costo, gasto o pasivo, o generado un ingreso.

Para la mayor parte de las transacciones que desarrolla el ente económico, la premisa de realización se cumple, bien porque se han recibido o entregado los bienes, porque se han recibido o prestado los servicios, porque se tiene el derecho a recibirlos o la obligación de entregarlos o prestarlos; sin embargo, existen algunos hechos económicos denominados contingencias de pérdida, en donde al momento del reconocimiento contable, el hecho económico aún no se ha realizado, pero las circunstancias que lo rodean hacen prever con alta probabilidad la futura ocurrencia del mismo. Por tanto, es necesario aplicar un criterio subjetivo prudente para reconocer o no el hecho económico.

2 La riqueza se determina por la estructura de activos, pasivos y patrimonio.

3 La rentabilidad se da por la combinación de ingresos menos costos y gastos. 
ACTIVOS | REVISTA DE LA FACULTAD DE CONTADURía PÚBLICA

La medición atiende a la forma como se asigna valor a una transacción. Usualmente, el valor de las transacciones se determina con base en valores reales descritos en los documentos de soporte, tales como facturas, escrituras, títulos valores, contratos, etc.; no obstante, algunas veces es necesario hacer mediciones estimadas que originan activos, pasivos, costos y gastos, en donde se requiere aplicar un criterio prudente para establecer de manera confiable el monto de las transacciones. Tal sería el caso de provisiones de activos, que generarán un gasto por provisiones, valorizaciones y desvalorizaciones de activos, pasivos estimados que generarán activos, costos y gastos estimados, y pasivos contingentes que generarán gastos estimados.

La asignación se refiere al traslado de parte del costo del activo a costos y gastos, para reconocer poco a poco el uso o consumo de los activos. El valor de la asignación periódica está afectado por el monto del activo; el método que se utilice para asignar inventarios, activos fijos, intangibles, y activos diferidos; las vidas útiles de activos fijos, intangibles, y activos diferidos; y los valores de salvamento de activos fijos.

La información tiene importancia relativa o es material, cuando su omisión o presentación errónea pueden influir en las decisiones económicas de los usuarios tomadas a partir de los estados financieros. Al tasar la materialidad, se evalúa la inclusión o exclusión de una transacción, o la corrección o no de un error, en un período contable ya cerrado, para efectos de emisión de estados financieros, dependiendo de la cuantía de la partida omitida, o del error de evaluación en su caso, juzgados siempre dentro de las circunstancias particulares de la omisión o el error.

Frente a este tema, el contador debe evaluar en qué grado se afecta la razonabilidad de los estados financieros tomados en conjunto, al incluir, excluir, corregir o no una partida. Las normas de auditoría de general aceptación hablan de cifras no importantes si equivalen hasta el 5\% de otra cifra, cifras a ser evaluadas si están entre el 5.1\% y el 10\%, y cifras importantes cuando equivalen a más del $10 \%$.

El criterio prudente implica aplicar juicios basados en datos históricos, experiencias anteriores e información disponible, para no subestimar pasivos, costos y gastos, y no sobrestimar ingresos y activos. El D. R. 2649/93, artículo 17, "Prudencia", describe que "Cuando quiera que existan dificultades para medir de manera confiable y verificable un hecho económico realizado, se debe optar por registrar la alternativa que tenga menos probabilidad de sobrestimar los activos y los ingresos, o de subestimar los pasivos y los gastos”. 
Asimismo, en el marco conceptual para la preparación y presentación de estados financieros emitido por el International Accounting Standard Board, se indica en el párrafo 37:

...la prudencia se refiere a que los elaboradores de estados financieros tienen que enfrentarse con las incertidumbres, que inevitablemente rodean muchos acontecimientos, como la recuperabilidad de los saldos dudosos, la vida útil probable de la Propiedad, Planta y Equipo, el número de reclamaciones por garantía posventa que se puedan recibir, etc. Tales incertidumbres deben ser reconocidas en los estados financieros aplicando un criterio prudente.

Definen como criterio prudente, "la inclusión de un cierto grado de precaución, al hacer los juicios necesarios para realizar las estimaciones requeridas bajo condiciones de incertidumbre, de tal manera que los activos y los ingresos no se sobrevaloren, y los pasivos, los costos y los gastos no se subvaloren".

\section{Criterio prudente en la valoración de activos}

En la medición de los activos, se presentan varios casos: los factores que afectan la porción del activo que se traslada a costos y gastos, las posibles pérdidas del beneficio económico futuro de los activos, y las ganancias o pérdidas no realizadas.

\section{Factores que afectan la porción del activo que se traslada a costos y gastos}

El costo del activo, la vida útil, el valor de salvamento, y el método de asignación, son los factores que afectan la porción del activo que se traslada mes a mes a costos y gastos para reconocer el uso o consumo de los mismos en desarrollo de las operaciones; por tanto, se requiere de un juicio prudente al estimar o escoger los mismos, los cuales deben basarse en conceptos de expertos, experiencias anteriores, especificaciones tecnológicas, etc.

El costo del activo se puede determinar de manera real o de forma estimada, pues es posible recibir el bien pero desconocer el monto del mismo, lo que implicaría el reconocimiento de un activo y de un pasivo estimado. Un caso específico es la recepción de materias primas y materiales de empaque en las bodegas sin la recepción de la factura; el monto se puede establecer con base en los precios establecidos en las órdenes de compra. 
ACTIVOS | REVISTA DE LA FaCULTAD DE CONTADURía PÚblica

La vida útil es el período contable durante el cual se espera obtener beneficio económico del activo. Si se establece una vida útil contable mayor, en el momento en que el activo se consuma o no se use, deja de contribuir a la generación de ingresos; sin embargo, se sigue trasladando parte de su costo a los gastos y/o los costos, disminuyendo las utilidades del ente económico. Por el contrario, si se establece una vida útil contable menor, habrá algunos períodos en que los activos ayuden a la generación de ingresos, sin que ello implique un costo o gasto asociado por depreciación, amortización o agotamiento.

Ejemplo de ello son los equipos de computación, que por los constantes cambios de tecnología pueden quedar rápidamente obsoletos; si se determina una vida útil mayor, es posible que se dejen de utilizar y quede una cifra por depreciar al final, la cual afectará el resultado del ejercicio.

El valor de salvamento de los activos fijos, equivale al monto estimado de venta de un activo fijo una vez termine su vida útil contable. La base de depreciación, amortización o agotamiento (costo del activo - valor de salvamento) es el monto que mes a mes se depreciará, amortizará o agotará, teniendo en cuenta el factor que determina la vida útil. Si el valor de salvamento es superior a la cantidad por la cual el mismo se venderá, se dejará de trasladar a costos y gastos una parte del costo del activo que se usó, consumió, o agotó; por el contrario, si el valor de salvamento es inferior a la cantidad por la cual el mismo se venderá, durante el tiempo de vida útil se trasladará a costos y gastos un mayor valor del activo.

El método de asignación es la forma sistemática como se determina de manera periódica el valor del costo del activo que se trasladará a costos y gastos. La escogencia de uno u otro método afectará año tras año los niveles de riqueza, de renta y de impuesto sobre la renta.

En el caso de los inventarios se puede optar por métodos como "PEPS" (primeros en entrar primeros en salir), "UEPS" (últimos en entrar primeros en salir), promedio ponderado, identificación específica, entre otros. En una economía de precios al alza, si se escoge el método "PEPS" se trasladará a costos los precios más bajos y en inventarios quedarán los precios más altos, por ende de menor costo, mayor utilidad y mayor impuesto de renta. Si se elige el método "UEPS" se trasladará a costos los precios más altos y en inventarios quedarán los precios más bajos, por ende de mayor costo, menor utilidad y menor impuesto de renta. En tanto que si se escoge método promedio ponderado, los cambios en precios van afectando los inventarios, los costos, la utilidad y el impuesto de renta. 
Tratándose de activos fijos, intangibles y activos diferidos, se pueden escoger métodos para depreciar, amortizar y agotar, tales como línea recta, unidades producidas, suma de los dígitos, saldos decrecientes, entre otros. Si se utiliza el método de línea recta, sin importar el aporte del activo a la generación del ingreso, el costo del activo se traslada a costos y gastos de manera similar durante toda su vida útil. Si se recurre al método de unidades producidas, el costo por depreciación, amortización o agotamiento, estará asociado directamente con la generación del ingreso; si se asigna con los métodos de suma de los dígitos y de saldos decrecientes, en los primeros años de vida útil del activo se asignarán mayores valores por depreciación y amortización que en los últimos años.

\section{Posibles pérdidas del beneficio económico futuro de los activos}

Los beneficios económicos futuros están referidos a la capacidad que tienen los activos de convertirse en dinero, usarse, explotarse, consumirse o venderse. El disponible se utiliza como medio de pago; las inversiones en general se convierten en dinero; las cuentas a cobrar se convierten en efectivo o minimizan futuros pasivos; los inventarios se consumen, se transforman, o se venden; los activos fijos se usan, se explotan o se consumen; los activos intangibles se explotan; y los activos diferidos se consumen.

Dado que el activo conserva sus características mientras mantenga el beneficio económico futuro, las posibles pérdidas del beneficio futuro se deben determinar de manera sensata, teniendo en cuenta los conceptos emitidos por asesores jurídicos, por expertos, el historial de la compañía, las condiciones del mercado, etc.

Si la probable pérdida se estima por encima del valor futuro real, se subvalora el activo y se incrementan los gastos del período por concepto de provisiones; por el contrario, si la posible pérdida se estima por debajo del futuro valor real, se sobrestima el valor del activo y se afectan en menor proporción los gastos del período por provisiones. Estas dos situaciones influyen en los niveles de riqueza y de rentabilidad.

Un caso específico es la probabilidad de cobranza de la cartera, cuando existen cuentas a cobrar vencidas entre uno y más de 360 días, en donde se establecen porcentajes de provisión para prever posibles pérdidas de acuerdo a la experiencia de la compañía y del sector. Si la provisión es muy baja, se dejan de reconocer gastos operacionales; por ende, el nivel de utilidad neta es alto y se pueden distribuir utilidades no reales que descapitalizan la compañía. 
ACTIVOS | REVISTA DE LA FaCULTAD DE CONTADURía PÚblica

\section{Ganancias o pérdidas no realizadas}

La determinación de los montos de ganancias o pérdidas no realizadas por valorizaciones o desvalorizaciones de activos fijos, inversiones y otros activos, implican un juicio razonable basado en conceptos de expertos en avaluación de bienes corporales e incorporales, ya que si el monto del avalúo se calcula por encima de la realidad económica, contablemente se muestra un mayor valor de activos y, por ende, un mayor valor de patrimonio, lo que indirectamente muestra a los usuarios de la información que hay solvencia económica, un mayor crecimiento de activos, etc. Por el contrario, si el monto del avalúo se calcula por debajo de la realidad económica, contablemente se presenta un menor valor de activos $\mathrm{y}$, por ende, un menor valor de patrimonio, lo que indirectamente revela a los usuarios de la información que hay menor solvencia económica, menor crecimiento de activos, etc.

\section{Criterio prudente en la valoración de pasivos}

En referencia a los pasivos existen dos casos: pasivos contingentes que afectan gastos, y pasivos estimados por bienes y servicios recibidos cuyo monto final se desconoce, que pueden afectar activos, costos y gastos.

\section{Pasivos contingentes}

Las normas contables colombianas descritas en el D. R. 2649/93, artículo 52 -"Provisiones y contingencias", hacen referencia a la contabilización de las provisiones para cubrir contingencias de pérdida probables. Este artículo aporta la siguiente definición:

Una contingencia es una condición, situación o conjunto de circunstancias existentes, que implican duda respecto de una posible ganancia o pérdida por parte de un ente económico, duda que se resolverá cuando uno o más eventos futuros ocurran o dejen de ocurrir. Las contingencias pueden ser probables, eventuales o remotas.

Las contingencias pueden ser de ganancia o de pérdida. En cuanto a las contingencias de ganancia, la prudencia contable aconseja no reconocer ingresos que no se hayan realizado, ya que si se reconoce el ingreso, se crea un derecho de cobro que probablemente no se ejecute, se causa un gasto y una obligación de pago de impuestos, sobre ingresos que posiblemente no se reciban; además, se pueden distribuir ganancias sobre ingresos que 
eventualmente no se perciban. Uno de estos ingresos corresponde a las futuras indemnizaciones por demandas interpuestas, ya que se desconoce el resultado de la demanda y el tiempo en el cual se reciban las mismas; otro caso se refiere a los intereses moratorios por recibir, en donde se desconoce si el tercero pagará el monto y cuando lo hará.

La prudencia contable recomienda reconocer contingencias de pérdida, cuando las circunstancias actuales muestran con alta probabilidad la ocurrencia futura de un pasivo; por ejemplo, las demandas interpuestas por terceros cuando se estima que las mismas van a ser ganadas por estos, el incumplimiento de contratos con cláusulas de penalización, el incurrir en cuasidelitos ${ }^{4}$, etc.

En estos casos, el criterio prudente implica reconocer un posible gasto y una potencial obligación de pago, teniendo en cuenta los conceptos de los abogados, los montos de las sanciones estipuladas en los contratos, etc. En el caso de una demanda, el posible valor a cancelar por indemnización debe tener en cuenta las pretensiones económicas del demandante, las posibilidades de negociación según el criterio de los abogados que llevan el caso, los términos estipulados en el contrato, etc.

\section{Pasivos estimados}

Existen dos clases de pasivos estimados: los primeros nacen por la recepción de un bien o de un servicio que origina un pasivo cuyo pago será cercano a la fecha de recepción (por ejemplo, los servicios públicos domiciliarios); y los segundos se van acumulando en el tiempo hasta que llega la hora del pago (por ejemplo, las prestaciones sociales, las pensiones de jubilación, etc.).

El primer tipo de pasivo estimado se presenta cuando se ha recibido un bien o un servicio, pero se desconoce el monto total del mismo. La norma contable de causación solicita reconocer la obligación futura de pago, ya que la misma se ha realizado al recibir el bien o el servicio a satisfacción. Sin embargo, para poder reconocer, además de la realización se requiere de una medición razonable; por tanto, es necesario estimar el valor de la obligación de manera prudente teniendo en cuenta el historial de consumos anteriores, los precios de referencia, los posibles incrementos de precios y consumos, entre otros. 
ACTIVOS | REVISTA DE LA FACULTAD DE CONTADURÍA PÚ́blica

Los casos más frecuentes son las obligaciones por servicios públicos domiciliarios. Una aplicación concreta de lo anterior es la factura de acueducto y alcantarillado que se recibe, por ejemplo, el 12 de mayo, para ser cancelada el 26 de mayo, en donde se factura el período comprendido entre el 1 de marzo y el 30 de abril por $\$ 1.200 .000$. Contablemente se causa una porción del pasivo en el mes de marzo y otra en el mes de abril, y en el mes de mayo, cuando se recibe la factura, se ajusta el pasivo, el costo, y el gasto de acuerdo al consumo real.

El segundo tipo de pasivo estimado se presenta cuando la realización de algunos hechos económicos conlleva indirectamente la conformación de un futuro pasivo, pero se desconoce el monto total del mismo. La norma contable de causación solicita reconocer la obligación futura de pago, ya que la misma se ha realizado al entregar el bien (garantías), o el recibo del servicio a satisfacción (mano de obra); no obstante, para poder reconocer se requiere de una medición razonable, entonces es necesario estimar el valor de la obligación de manera prudente teniendo en cuenta los factores que influyen en la misma.

Para el caso de las prestaciones sociales, las primas de servicio se pagan en los meses de junio y diciembre del mismo año, los intereses sobre las cesantías se cancelan en el mes de enero del siguiente año, y las cesantías se pagan en el mes de febrero del año siguiente. Dado que las prestaciones se determinan con base en los devengos de los trabajadores acumulados de manera anual y semestral, es necesario establecer mes a mes una parte proporcional de dicho pasivo, de tal manera que se logre una adecuada asociación ente los ingresos por ventas, y los costos y gastos necesarios para su generación. Es así como se reconocen provisiones para prestaciones sobre los devengos mensuales con su correspondiente efecto en costos y gastos, que pueden variar entre el $8.33 \%$ al $9 \%$ para cesantías y primas y entre el $4.17 \%$ y el $5 \%$ para intereses sobre cesantías.

En el caso de garantías sobre el funcionamiento de productos, que por lo regular varían entre tres meses y tres años, las compañías usualmente establecen mes a mes un porcentaje sobre las ventas, para ir acumulando el posible pasivo que atienda los reclamos por garantías. El criterio prudente deberá incluir factores como cantidades de reclamaciones, montos de reclamaciones en años anteriores, etc.

\section{Conclusión}

Las diferentes premisas para el reconocimiento de los hechos económicos están afectadas por un criterio prudente: la realización por la futura ocurrencia de pasivos contingentes; la medición por la forma como se determina el valor de provisiones para activos, el valor 
de las valorizaciones o desvalorizaciones, el valor de los pasivos estimados, y el valor de los pasivos contingentes; la asignación por el método que se utilice para asignar, por la determinación de vidas útiles de activos, y por la forma como se determinan los valores de salvamento de los activos; y la materialidad por la subjetividad del contador y su equipo de colaboradores para reconocer o corregir una transacción.

La aplicación del criterio prudente debe ser tal que no induzca a la sobre-valoración de activos e ingresos ni a la sub-valoración de pasivos, costos y gastos y, por ende, no lleve a los administradores de las compañías a distribuir utilidades inexistentes por falta del reconocimiento de provisiones para activos o pasivos estimados, y a los usuarios de la información a tomar decisiones equívocas, especialmente en el análisis de las contingencias y valoraciones de hechos económicos.

Por último, para que la contabilidad sea razonable, se requiere que el contador y su equipo de colaboradores hagan uso de un criterio prudente y utilicen las herramientas adecuadas para que las transacciones se reconozcan de manera confiable. 